\section{Control de la resistencia a los antimicrobianos en los hospitales: control de las infecciones y uso de antibióticos}

En los hospitales, la resistencia a los antimicrobianos es determinada por problemas de falta de higiene, por la presión selectiva creada por el uso excesivo de los antibióticos y por los elementos genéticos móviles codificadores de mecanismos de resistencia bacteriana.

En una encuesta realizada en cuatro centros médicos de los Estados Unidos de América, 85\% de los 424 médicos encuestados consideraron que la resistencia a los antimicrobianos es un importante problema nacional y 55\% declararon que la resistencia era un problema para sus enfermos (6). En este artículo se aborda el problema de la higiene y de la presión de los antibióticos.

En una encuesta reciente, $45 \%$ de los médicos entrevistados consideraron que las malas prácticas de lavado de las manos constituyen una importante causa de resistencia a los antimicrobianos en los hospitales. El lavado de las manos es una de las medidas relacionadas con el control de las infecciones que peor se cumple. A ello contribuyen el tiempo que consume y los efectos adversos cutáneos del lavado repetido. Las friegas con alcohol pueden superar ambos problemas, pero la adherencia a esta práctica requiere refuerzos educativos continuos, un control de su cumplimiento y retroalimentación del personal. Con campañas agresivas de este tipo se pueden conseguir tasas de higiene de las manos del $60 \%$ al $80 \%$, suficientes para patógenos poco frecuentes que solo colonicen una pequeña proporción de pacientes. Sin embargo, cuando la presión de colonización es mayor debido a la existencia de un mayor número de pacientes colonizados, estas cifras pueden ser insuficientes. Por ejemplo, cuando el 30 a 50\% de los pacientes están colonizados por enterococos resistentes a la vancomicina incluso descuidos ocasionales con el lavado de las manos pueden ser suficientes para mantener la transmisión cruzada.

Una medida para contrarrestar el problema de la presión de colonización es el uso de guantes desechables durante el contacto con los pacientes y su entorno. En un estudio, la tasa de diarrea nosocomial por Clostridium difficile fue tres veces menor en plantas con "uso universal de guantes" que en las plantas de control (16). En un estudio sobre los enterococos resistente a la vancomicina, 39\% del personal tenía los guantes contaminados por estos microorganismos, incluso después de un breve contacto con pacientes infectados o colonizados; la contaminación de las manos del personal disminuyó en un $71 \%$ con el uso de guantes.

La presión de los antibióticos puede ser más susceptible de intervención que las prácticas higiénicas. Para simplificar la prescripción de antibióticos, la mayoría de los hospitales utilizan formularios "cerrados" que reducen las opciones de prescripción, generalmente a uno o dos fármacos por clase de antibióticos. Las pautas clínicas pueden mejorar el empleo de los antibióticos, especialmente si se realizan auditorías y se les proporciona retroalimentación a los médicos. Las intervenciones más eficaces relacionadas con los antibióticos han sido los programas de restricción y las recetas informatizadas.

Las restricciones al uso de antibióticos han sido especialmente eficaces para reducir los costes y el excesivo uso empírico de los antibióticos de amplio espectro.

La intervención más reciente en la prescripción de antibióticos ha sido su empleo rotatorio, que tiene mayores probabilidades de ser eficaz durante períodos de tiempo limitados y en ambientes cerrados, como las unidades de cuidados intensivos.

El control de la resistencia a los antibióticos requiere la implementación de varias estrategias: vigilancia continua de la resistencia; tipificación molecular de los aislados; empleo de controles higiénicos para reducir la propagación de cepas clonales y de controles de antibióticos para limitar la propagación de las cepas policlonales, y apoyo administrativo. (Weinstein RA. Controlling antimicrobial resistance in hospitals: infection control and use of antibiotics. Emerg Infect Dis 2001;7:188-192)

\section{Factores socioeconómicos y ambientales protectores o de riesgo frente a la malaria grave en Tailandia}

Todavía no se conocen bien los factores que predisponen a la malaria grave, pero se cree que entre ellos hay factores relacionados con el huésped y con el parásito. En este estudio transversal, los autores analizan los factores socioeconómicos y ambientales que predisponen a la malaria grave o protegen frente a ella. El estudio se realizó en Tailandia entre febrero y diciembre de 1998. Participaron 46 
casos de malaria grave, 72 de malaria no grave con gran biomasa parasitaria y 40 con malaria leve. Los casos graves se definieron por la presencia de un frotis de sangre positivo para Plasmodium falciparum, con alteración de la consciencia (puntuación de Glasgow del coma $\leq 11$ ) o creatinina sérica $>3 \mathrm{mg} / \mathrm{dl}$. Los casos no graves con gran biomasa parasitaria se definieron como aquellos que tenían parasitados más del 5\% de los eritrocitos, que presentaban recuentos de parásitos $>200$ 000/dl o que tenían esquizontes circulantes, pero que no cumplían ninguno de los criterios de malaria grave antes mencionados. A su vez, los pacientes con malaria leve presentaban frotis de sangre positivos para P. falciparum, pero no cumplían ninguno de los criterios antes mencionados y no tenían ictericia.

Todos los pacientes fueron entrevistados al cuarto día del ingreso para obtener datos acerca de la localización y duración de la residencia, del número de personas en la casa, de las medidas antivectoriales aplicadas (mosquiteros, repelentes fumigación de la casa con DDT), los ingresos, el nivel educacional, el origen étnico, las creencias sobre la gravedad de la malaria, los hábitos de automedicación, la distancia a los servicios sanitarios, la frecuencia de actividades forestales y la duración de los síntomas antes del ingreso. Regularmente, también se efectuaron recuentos hematológicos, pruebas bioquímicas sanguíneas y exámenes de heces. El estado nutricional se valoró en función del índice de masa corporal y se consideraron malnutridos los pacientes con valores inferiores a $18,5 \mathrm{~kg} / \mathrm{m}^{2}$.

En total se estudiaron 158 pacientes con malaria por $P$. falciparum. De los 46 con malaria grave, 4 tenían insuficiencia renal aguda, 12 tenían insuficiencia renal aguda y malaria cerebral, y 30 tenían malaria cerebral. La parasitemia mediana fue de 95 790/mL (rango intercuartílico: 30000 a 290 000/ $\mathrm{mL}$ ) para los casos graves, de $79000 / \mathrm{mL}(23000 \mathrm{a}$ $205000 / \mathrm{mL}$ ) para los casos de malaria no grave con gran biomasa parasitaria, y de $8100 / \mathrm{mL}$ (790 a $32400 / \mathrm{mL}$ ) para los casos de malaria leve.

En los modelos de regresión logística escalonada se incluyeron las siguientes variables: duración de los síntomas, edad, origen étnico, ingresos, uso de mosquiteros, uso de repelentes, automedicación antimalárica, fumigación de la casa con DDT, duración de la residencia, antecedentes personales de malaria, helmintiasis, índice de masa corporal y volumen corpuscular medio. De estos modelos se eliminaron las variables cuyo valor $P$ fue $<0,15$.

Al comparar los casos de malaria grave y leve, la mayor duración de la residencia y el empleo de medicaciones antimaláricas se asociaron a la protección frente a la malaria grave, con razones de los productos cruzados (odds ratio: OR) ajustadas de 0,36 (intervalo de confianza del 95\%: 0,09 a 0,83) y
0,08 (intervalo de confianza del 95\%: 0,009 a 0,84), respectivamente. A su vez, al comparar los casos de malaria grave con los de malaria no grave con gran biomasa parasitaria, las helmintiasis y el bajo índice de masa corporal también se asociaron a un significativo efecto protector, con OR ajustadas de 0,24 (intervalo de confianza del 95\%: 0,07 a 0,78) y 0,11 (intervalo de confianza del 95\%: 0,02 a 0,58). Incluyendo todas las variables en el modelo de regresión lineal escalonada se obtuvieron resultados similares.

A juicio de los autores, estos resultados indican que la inmunidad específica y la automedicación antimalárica están implicadas en el control de la multiplicación del parásito, mientras que las helmintiasis y la malnutrición afectan de forma más específica a la patogénesis de la malaria grave. (Nacher M, Singhasivanon P, Vannaphan S, Treeprasertsuk S, Phanumaphorn M, Traore B, et al. Socioeconomic and environmental protective:risk factors for severe malaria in Thailand. Acta Tropica 2001; 78:139-146)

\section{Estudio de casos y controles sobre el programa de vacunación acelerada del Reino Unido y la muerte súbita inesperada en la infancia}

La edad con la que los niños reciben sus primeras vacunas coincide con la edad de máxima incidencia del síndrome de muerte súbita del lactante, lo cual ha llevado a pensar que podría haber una relación. Durante los últimos 20 años, algunos informes esporádicos y algunos estudios de casos y controles mostraron una posible asociación, pero otros han llegado a la conclusión contraria, y uno señaló la posibilidad de que un programa de vacunación acelerada contribuyera directamente a reducir estas muertes. Todos estos estudios tienen la debilidad de que pueden estar sesgados por factores de confusión residuales.

En 1990 se aceleró el programa nacional de vacunación del Reino Unido, pasándose a administrar la vacuna contra el tétanos, la difteria y la tos ferina y la vacuna antipoliomielítica oral a los 2, 3 y 4 meses, en vez de a los 3, 5 y 9 meses. En 1992 se empezó a administrar también la vacuna contra el Haemophilus influenzae b. Los autores de este informe han realizado un amplio estudio de casos y controles sobre la muerte súbita inesperada del lactante después del cambio del programa de vacunación y de la reducción de la tasa de muerte súbita del lactante a principios de la década de los 90. En este informe se analiza la posible existencia de una relación temporal entre el programa de vacunación acelerada y el momento de la muerte.

Para ello se realizó un amplio estudio de casos y controles basado en la población en el que se in- 
tentaron incluir todos los casos de muerte súbita inesperada, tanto explicados como inexplicados, de niños de una semana a un año de edad que tuvieron lugar en una población de 17,7 millones de personas entre febrero de 1993 y marzo de 1996. Por cada caso se seleccionaron cuatro controles emparejados por edad, localidad y hora del sueño.

Durante los 3 años hubo más de 470000 nacimientos en el área estudiada y 456 muertes súbitas inesperadas de lactantes, de las cuales 363 fueron atribuidas al síndrome de muerte súbita del lactante (SMSL). Se realizaron entrevistas con los padres de 325 niños cuya muerte se atribuyó al SMSL $(90 \%)$, de 72 de los 93 niños con muertes explicadas $(77 \%)$ y de los controles. Se obtuvieron datos sobre la vacunación en 93\% (303/325) de los lactantes cuya muerte se atribuyó al SMSL, 90\% (65/72) de los lactantes con muerte explicada y 95\% (1 515/ 1588 ) de los controles.

Entre los 72 casos de muerte explicada, las principales causas de muerte fueron las infecciones no identificadas $(46 \%)$, los accidentes $(15 \%)$, las malformaciones congénitas $(14 \%)$ y las lesiones no accidentales (13\%).

Poco menos de la mitad (149/303) de los niños con SMSL habían iniciado o completado el programa de vacunación, en comparación con dos tercios (822/1 234) de los controles: razón de los productos cruzados (odds ratio: OR) ajustada por el emparejamiento de 0,23 (intervalo de confianza del 95\%: 0,14 a 0,37). La captación del programa, basada en los niños de 3 o más meses de edad, fue del $93 \%$ (638/688) en los controles y del 79\% (116/146) en los casos índice. Después de controlar los efectos de múltiples factores de confusión (edad del lactante y de la madre, grupo socioeconómico, cambio de domicilio en el año anterior, número de niños en la familia, peso al nacer, edad gestacional, ingreso en la unidad de cuidados especiales neonatales, ingreso hospitalario, puntuación de Apgar a los 5 minutos y antecedentes de acontecimientos potencialmente mortales), la captación del programa de vacunación siguió estando estrechamente asociada a un menor riesgo de SMSL (OR multivariada de 0,45; intervalo de confianza del $95 \%$ : 0,24 a 0,85 ). No obstante, cuando también se controló el efecto de factores de riesgo importantes relacionados con el sueño, como el decúbito prono o la presencia de ropa de cama sobre la cabeza, la diferencia dejó de ser significativa (OR: 0,67; intervalo de confianza del 95\%: 0,31 a 1,43).

De los niños que fallecieron por causas explicadas, 54\% (35/65) habían iniciado o completado el programa de vacunación, en comparación con el 61\% (172/281) de los controles (OR univariada: 0,51; intervalo de confianza del 95\%: 0,21 a 1,26).
La edad mediana (rango intercuartílico) a la que se administró la primera vacuna fue de 61 (56-71) días para los niños con SMSL y de 59 (36-63) días para los controles. El intervalo de tiempo mediano entre la vacunación y la muerte fue de 27 (16-68) días, similar al intervalo de 29 (13-70) días entre la vacunación y la entrevista en el grupo de control. Un 5\% (7/149) de los niños vacunados con SMSL habían sido vacunados 48 horas antes de la muerte, igual que un 5\% (41/822) de los controles vacunados lo habían sido 48 horas antes del sueño de referencia.

De acuerdo con los resultados obtenidos, los autores concluyen que: 1 ) incluso después de tener en cuenta múltiples factores de confusión, la vacunación no conduce a la muerte súbita inesperada del lactante y la dirección de la relación es hacia la protección y no hacia el riesgo, y 2) el programa de vacunación acelerada del Reino Unido no se asocia a la muerte súbita inesperada del lactante, explicable o no. (Fleming PJ, Blair PS, Platt MW, Tripp J, Smith IJ, Golding J. The UK accelerated immunisation programme and sudden unexpected death in infancy: case-control study. BMJ 2001;322: 822-825)

\section{Parto pretérmino: ensayo clínico aleatorizado comparativo del tratamiento domiciliario frente al tratamiento hospitalario}

El parto pretérmino afecta a cerca de un $10 \%$ de los embarazos y es la causa más importante de nacimiento prematuro. Las mujeres con parto pretérmino suelen ingresar en el hospital para detener las contracciones uterinas y muchas permanecen hospitalizadas hasta el parto. Aunque hay muchos defensores del tratamiento hospitalario, no se ha demostrado que mejore los desenlaces perinatales en mujeres con embarazos no gemelares de alto riesgo. Dado el aumento de los costes sanitarios, los médicos se han visto forzados a adelantar el alta de las pacientes, a menudo sin incluirlas en programas especiales de seguimiento. Los autores de este estudio realizaron un ensayo clínico aleatorizado para comparar los desenlaces maternos y neonatales obtenidos con la atención hospitalaria y la atención domiciliaria en mujeres que habían sido ingresadas en el hospital por parto pretérmino. Los desenlaces neonatales primarios analizados fueron la edad gestacional y el peso al nacer; como medidas secundarias se analizaron las tasas de parto pretérmino, la duración de la hospitalización neonatal y el número de ingresos en la unidad de cuidados intensivos neonatales. A su vez, los desenlaces maternos analizados fueron el número de hospitalizaciones y su duración. 
Después de haber recibido tratamiento hospitalario por un episodio agudo de parto pretérmino, las participantes fueron asignadas aleatoriamente a un grupo que siguió siendo atendido en el hospital o a un grupo que fue atendido en el domicilio, donde fueron monitorizadas por enfermeras. Las 250 mujeres elegibles cumplían los siguientes requisitos: edad $\geq 18$ años, residencia a menos de $50 \mathrm{~km}$ del hospital, edad gestacional entre 20 y 35 semanas, ausencia de antecedentes de parto pretérmino y primer episodio de parto pretérmino y de ingreso hospitalario por este motivo. Se excluyeron las mujeres con más de una complicación del embarazo en el momento de la asignación aleatoria, con dilatación cervical $>4 \mathrm{~cm}$ y borramiento $>80 \%$ o con diagnóstico de muerte intrauterina o malformación fetal.

Para determinar hasta qué punto las mujeres se acercaban al objetivo de conseguir un parto a término, se utilizaron las siguientes fórmulas, en las que el partos a término se definió como el que tuvo lugar a las 38 o a las 40 semanas de gestación: porcentaje de partos a término a las 38 semanas $=100 \times$ (edad gestacional en el momento del parto - edad gestacional en el momento de la asignación aleatoria)/(266 días - edad gestacional en el momento de la asignación aleatoria), y porcentaje de partos a término a las 40 semanas $=100 \times($ edad gestacional en el momento del parto - edad gestacional en el momento de la asignación aleatoria)/(280 días edad gestacional en el momento de la asignación aleatoria). La muestra final de 250 mujeres proporcionó una potencia del 89\% para detectar una diferencia de más de una semana entre las edades gestacionales medias de uno y otro grupo. Los datos fueron analizados por intención de tratar.

Los dos grupos fueron comparables con respecto a las siguientes características sociodemográficas: edad materna, nivel educacional, estado civil, ingresos familiares y empleo durante el embarazo. Asimismo, fueron comparables con respecto a las siguientes características obstétricas basales: edad gestacional, borramiento y dilatación en el momento de la asignación aleatoria, número de embarazos, partos y abortos, número de hijos vivos y proporciones de casos tratados con tocolíticos y betametasona, y método de inicio, duración del parto y método del parto.

No hubo diferencias significativas entre los grupos con respecto a la edad gestacional en el momento del parto (riesgo relativo [RR]: 0,96; intervalo de confianza del 95\% [IC95\%]: 0,73 a 1,28) ni al peso al nacer (1500 g o > $1500 \mathrm{~g}$; RR: 1,968: IC95\%: 0,52-7,50). El número de niños ingresados en la unidad de cuidados intensivos neonatales también fue aproximadamente el mismo en los dos grupos, y lo mismo ocurrió con el número de niños que tuvieron múltiples problemas de salud.

No hubo diferencias significativas entre los dos grupos con respecto a la edad gestacional media en el momento del parto (domicilio: 37,52 semanas y hospital: 37,50 semanas), al peso medio al nacer (domicilio: 2974 g, hospital: $3020 \mathrm{~g}$ ), a la proporción de niños nacidos antes del término ni a la duración media de la estancia del recién nacido en el hospital, en la unidad de cuidados intensivos neonatales o en la unidad de cuidados intermedios. Con respecto a los desenlaces maternos, la duración media del primer ingreso hospitalario fue significativamente menor en el grupo domiciliario (3,8 días) que en el hospitalario (6,1 días), y lo mismo ocurrió con la duración media de todas las estancias maternas en el hospital: 3,7 frente a 5,0 días, respectivamente. Las dos medidas porcentuales del parto a término (a las 38 y a las 40 semanas) no presentaron diferencias estadísticamente significativas entre los grupos.

De acuerdo con estos resultados, la atención a domicilio constituye una alternativa eficiente y aceptable a la atención hospitalaria en mujeres con parto pretérmino. Los autores creen que la atención hospitalaria se debería reservar para las mujeres con trastornos graves que necesiten una intensa vigilancia médica. (Goulet $C$, Gévry $H$, Lemay $M$, Gauthier RJ, Lepage L, Fraser W, et al. A randomized clinical trial of care for women with preterm labour: home management versus hospital management. CMAJ 2001;164:985-91) 